\title{
Animal and Plant Species in Antagonistic and Mutualistic Networks, a Multispecies Mean Field Model
}

\author{
Gilberto Corso $^{1 *}$, Claudia P. T. Cruz ${ }^{2}$, Adriana M. de Almeida ${ }^{3}$ \\ ${ }^{1}$ Departamento de Biofisica e Farmacologia, Centro de Biociências, Universidade Federal do Rio Grande do Norte, \\ Natal, Brazil \\ ${ }^{2}$ Escola de Engenharia e Ciências Exatas, Universidade Potiguar, Natal, Brazil \\ ${ }^{3}$ Departmento de Ecologia, Centro de Biociências, Universidade Federal do Rio Grande do Norte, Natal, Brazil \\ Email: *corso@cb.ufrn.br
}

How to cite this paper: Corso, G., Cruz, C.P.T. and de Almeida, A.M. (2017) Animal and Plant Species in Antagonistic and Mutualistic Networks, a Multispecies Mean Field Model. Open Journal of Ecology, 7, 211-221.

https://doi.org/10.4236/oje.2017.73016

Received: February 8, 2017

Accepted: March 27, 2017

Published: March 30, 2017

Copyright (c) 2017 by authors and Scientific Research Publishing Inc. This work is licensed under the Creative Commons Attribution International License (CC BY 4.0).

http://creativecommons.org/licenses/by/4.0/

\begin{abstract}
We construct a dynamic system model of a web of $N_{A}$ animal species interacting with $N_{P}$ plant species using a system of coupled differential equations. The model has a parameter which represents the effect of animal on plant species that can be positive (mutualistic interaction) or negative (antagonistic interaction). We work a Multispecies Mean Field Model MMFM in which the full set of $N_{A}$ and $N_{P}$ variables are reduced to a couple of an average animal and plant species. We study the conditions for existence of the MMFM and relate the result to the difference between animal and plant species in the network. We compare our results with empirical data from pollinators (mutualistic) and herbivorous insect (antagonistic) networks. We conclude by combining analytical and empirical work that antagonistic networks present fewer animal species in relation to plant species than mutualistic ones.
\end{abstract}

\section{Keywords}

Ecological Interactions, Stability Complexity, Plant-Animals Ratio, Pollination, Herbivory

\section{Introduction}

Since the seminal paper [1], the stability complexity debate has evolved to a hot topic in theoretical ecology [2] [3]. From a mathematical perspective, the stability complexity problem can be posed in at least two different contexts: firstly, the traditional approach of population dynamics, a set of coupled differential equations representing the density of a set of many interacting species [1] [4] [5] 
[6]. In this framework, the main idea is to work the stability of the fixed points of the equations describing the time evolution of the populations [7]. On the other side, the stability complexity problem can be formulated using a more contemporary framework, namely the network theory [8] [9] [10]. In this approach, the focus is on the number, distribution, or assortativity of interactions, in one word: the pattern of connections in the network [11] [12].

In this work, we explore both mathematical approaches. Firstly, the coupled dynamic system is a flexible schema that permits a modeling of complex systems using phenomenological parameters such as the intrinsic growth rate of a population, its carrying capacity, or the interaction strength among species. All cited parameters can, in principle, be empirically estimated [13]. In addition, most empirical data available to compare with dynamic system models are records of food webs or pollinator networks which indicate the presence of interaction among a set of species. Confident time series records of populations interacting in a community are not available, but network inventories of interaction among species are common in the literature. We cite as examples web interaction inventories [14], pollinators flower networks [15] host parasite networks [16], and herbivory networks [17].

In this manuscript, we work with a dynamic multi-species model representing two different situations: mutualistic and antagonistic interactions. Pollination webs are formed by species which both profit from the interaction and then should be favored by adaptive forces. On the other hand, Herbivory (trophic) webs are formed by bipartite webs in which only herbivores profit. This is differently from pollination where in this case the plants are injured in the interaction and adaptive forces involved in the interaction are opposite to what found in pollination. We consider a set of $N_{A}$ animal species and a set of $N_{P}$ plant species that interact with each other, but do not interact among themselves. The objective of this paper is to construct a Multispecies Mean Field Model MMFM reducing the original $N_{A}+N_{P}$ equations to two equations that represent the average animal and plant species. In this approach, the set of coupled dynamic equations may have an intricate topology with many fixed points, limit cycles, strange attractors, or even chaos because of the intrinsic non-linearity [18]. The reduced MMFM, as we shall see, has just one single fixed point of interest. Despite the strong simplification in this methodology, we extract useful hints about network properties, espeacially regarding the network pattern related to the stability of interacting systems, a key point in the complexity stability debate. We are aware that the MMFM that we present here lacks a rigorous mathematical foundation. However, we persist in this theoretic field because of the promising results we have found until now.

In this paper, we consider a MMFM of a dynamic system of coupled Lotka Volterra like equations. The MMFM is a simplified two-dimension version of a multiple dimension system. The Lotka Volterra equations we deal with in our manuscript correspond to linear functional responses in the interaction term [19] [20], while we discuss more involved non-linear responses in the text. The 
analysis of the conditions for existence of the MMFM reveals some properties of the complex many dimensional dynamic network underlying the complex dynamics.

The main result of the cited paper of May [1] is that ecological networks are sparse. This conclusion is somewhat unexpected, we cite MacArthur that believed that large number of interaction will stabilize networks and not the opposite [21]. Our work also uses a coupled of differential equations to model ecological webs. In contrast, we use different technique and our main findings concern differences between antagonistic and mutualistic networks. Our manuscript shows a theoretical model that explains the difference between the number of plant and animals in herbivorous and pollinator networks. The rest of the paper is organized as follows: in Section 2, we present the full multiple dimension dynamic system used to describe the model and the associated MMFM. In Section 3 we separately compare the existing conditions for mutualistic and antagonistic networks with empirical data. Finally, we present our main findings in Section 4 and discuss the number of animal and plants in the community.

\section{Methodology}

This section is divided in two parts. Initially, we expose the biological data used to test the methodology, namely the set of binary and quantitative interaction networks. The second part of the methodology is devoted to describe the mean field model that is in the center of our analysis.

\subsection{The Biological Data Set}

In this work, we employed a set of quantitative webs extracted from the literature. We use a total of $n_{\text {total }}=44$ quantitative webs divided into two categories: pollinators $\left(n_{\text {poli }}=22\right)$ and herbivory $\left(n_{\text {herb }}=22\right)$. Pollinator webs are a typical example of a mutualistic networks formed by flowering plants and pollinator animal species, [22] [23]. Herbivory webs are a well studied example of antagonistic interaction, these networks are formed by herbivorous insect species and their respective host plants [24].

The empirical objects used in this work are ecological interaction webs which essentially are matrices of size $N_{A}$ versus $N_{P}$ corresponding to two groups of interacting animal and plant species, respectively. In the case of an interaction between species $i$ of the first set and species $j$ of the second the matrix element is $a_{i, j}=1$, otherwise $a_{i, j}=0$, for the binary case. In the more general case of quantitative matrices $a_{i, j}$ can assume any positive value. The connectivity of an animal species is defined as $k_{j}^{A}=\sum a_{i, j}$ and for a plant species $k_{i}^{P}=\sum a_{i, j}$. The empirical data used in this work is the same of the reference [25], where we show the full characteristic of the interacting networks. The herbivorous and pollinator webs used in this work are the totality of available webs in the literature at the time of the computation analysis. That means, we have not performed any exclusion criterion in the statistical analysis. The indices of the network we use are: the number of plant $N_{P}$ and animal species $N_{A}$, 
the total number of connections (without weight) among plant and animal species $L$, and the connectance $C=\frac{L}{N_{A} N_{P}}$.

\subsection{The Mean Field Approximation}

We employ a model that is a close extension of the multispecies model developed in the reference [26]. We extend the previous work to a model that encompasses both mutualistic and antagonistic interactions. The theory is based on a two-species model proposed in the reference [2] for the mutualistic case but it is even older in the antagonist situation [27]. The evolution of the animal $A_{j}$ and plant $P_{i}$ abundances in time is achieved by:

$$
\left\{\begin{array}{c}
\frac{\mathrm{d} A_{1}}{\mathrm{~d} t}=q_{1} A_{1}-T_{1} A_{1}^{2}+\sum_{i=1}^{N_{P}} \beta_{i 1} P_{i} A_{1} \\
\vdots \\
\frac{\mathrm{d} A_{N_{A}}}{\mathrm{~d} t}=q_{N_{A}} A_{N_{A}}-T_{N_{A}} A_{N_{A}}^{2}+\sum_{i=1}^{N_{P}} \beta_{i N_{A}} P_{i} A_{N_{A}} \\
\frac{\mathrm{d} P_{1}}{\mathrm{~d} t}=r_{1} P_{1}-S_{1} P_{1}^{2} \pm \sum_{j=1}^{N_{A}} \alpha_{1 j} P_{1} A_{j} \\
\vdots \\
\frac{\mathrm{d} P_{N_{P}}}{\mathrm{~d} t}=r_{N_{P}} P_{N_{P}}-S_{N_{P}} P_{N_{P}}^{2} \pm \sum_{j=1}^{N_{A}} \alpha_{N_{P} j} P_{N_{P}} A_{j}
\end{array}\right.
$$

where $P_{i}$ and $A_{j}$ represent the abundances of plant $i$ and animal $j ; r_{i}$ and $q_{j}$ are the growth rates of plants $i$ and $j$ while $S_{i}$ and $T_{j}$ are the respective intraspecific competition coefficients. In addition, $\alpha_{i j}$ and $\beta_{i j}$ are the per-capita effects of animal $j$ on plant $i$, and vice-versa. The positive signal of $\alpha_{i j}$ models a mutualistic dynamic, the effect of animals on plants is positive, so it implies in an increase in plant species abundance. On the other hand, a negative signal models an antagonistic interaction in which animal species restrict plant species growth.

We adopt a mean field approach that simplifies the model allowing further analytical insight. The reference [26] used a similar reduction technique to approximate modeling. Indeed, such reductions are common in theoretical ecology [27]. We assume that all plant species are equivalent, which means $r_{i}=r$, $T_{i}=T, \alpha_{i j}=\alpha$; and the same average equivalence is applied to animals: $q_{j}=q, T_{j}=T, \beta_{i j}=\beta$.

$$
\left\{\begin{array}{l}
\frac{\mathrm{d} A_{j}}{\mathrm{~d} t}=q A_{j}-T A_{j}^{2}+\beta \sum_{i=1}^{N_{P}} a_{i, j} P_{i} A_{j} \\
\frac{\mathrm{d} P_{i}}{\mathrm{~d} t}=r P_{i}-S P_{i}^{2} \pm \alpha \sum_{j=1}^{N_{A}} a_{i, j} P_{i} A_{j}
\end{array}\right.
$$

Moreover, we assume in the mean field approach that $a_{i, j} \rightarrow\left\langle a_{i, j}\right\rangle \rightarrow C$. This means, we average the individual elements of the adjacency matrix by the connectance of the matrix. In addition, we use the notation $P_{\text {tot }}=\sum_{i=1}^{N_{P}} P_{i}$ and $A_{\text {tot }}=\sum_{j=1}^{N_{A}} A_{j}$. The above equations become: 


$$
\left\{\begin{array}{l}
\frac{\mathrm{d} A_{j}}{\mathrm{~d} t}=q A_{j}-T A_{j}^{2}+\beta C P_{\mathrm{tot}} A_{j} \\
\frac{\mathrm{d} P_{i}}{\mathrm{~d} t}=r P_{i}-S P_{i}^{2} \pm \alpha C A_{\mathrm{tot}} P_{i}
\end{array}\right.
$$

We perform the equations' average for the set of animal and plant species in the following way. We add all $\frac{\mathrm{d} A_{j}}{\mathrm{~d} t}$ in the above equation and divide the result by $N_{A}$, the number of equations. Next, we introduce $\langle A\rangle=\sum A_{j} / N_{A}$; a similar estimation is performed on the equations of plant species. In addition, we assume that the approximations $\left\langle A^{2}\right\rangle \approx\langle A\rangle^{2}$ and $\left\langle P^{2}\right\rangle \approx\langle P\rangle^{2}$ are valid in the case differences inside the groups $A_{j}$ and $P_{i}$ are not significant. This condition is assumed in the mean field approximation. Following these estimations we obtain:

$$
\left\{\begin{array}{l}
\frac{\mathrm{d}\langle A\rangle}{\mathrm{d} t} \approx q\langle A\rangle-T\langle A\rangle^{2}+\beta C P_{\mathrm{tot}}\langle A\rangle \\
\frac{\mathrm{d}\langle P\rangle}{\mathrm{d} t} \approx r\langle P\rangle-S\langle P\rangle^{2} \pm \alpha C A_{\mathrm{tot}}\langle P\rangle
\end{array}\right.
$$

Finally, we use $P_{\text {tot }}=N_{P}\langle P\rangle$ and $A_{\text {tot }}=N_{A}\langle A\rangle$ to find:

$$
\left\{\begin{array}{l}
\frac{\mathrm{d}\langle A\rangle}{\mathrm{d} t} \approx q\langle A\rangle-T\langle A\rangle^{2}+\beta C N_{P}\langle P\rangle\langle A\rangle \\
\frac{\mathrm{d}\langle P\rangle}{\mathrm{d} t} \approx r\langle P\rangle-S\langle P\rangle^{2} \pm \alpha C N_{A}\langle A\rangle\langle P\rangle
\end{array}\right.
$$

We are aware that the mean field statement employed in the analytical treatment that reduces the dimension of the system from $N_{A} \times N_{P}$ to $2 \times 2$ is accompanied by a brutal loss of information [28] [29]. However, despite this strong assumption, the model produces theoretical estimations that encourage further development of the concept. We use the notation for the equilibrium solution: $\langle P\rangle=P^{*}$ and $\langle A\rangle=A^{*}$. With help of this notation we can write the equilibrium points of the MMFM as:

$$
\left\{\begin{array}{l}
\frac{\mathrm{d} A^{*}}{\mathrm{~d} t}=q A^{*}-T A^{* 2}+\beta C N_{P} P^{*} A^{*}=0 \\
\frac{\mathrm{d} P^{*}}{\mathrm{~d} t}=r P^{*}-S P^{* 2} \pm \alpha C N_{A} P^{*} A^{*}=0
\end{array}\right.
$$

There are four solutions for this pair of coupled system: the trivial null solution $(0,0)$, a solution with absence of plants $(0, q / T)$, another with absence of animals $(r / s, 0)$ and a non-trivial solution with $A^{*}$ and $P^{*}$ positives given by:

$$
\begin{aligned}
& A^{*}=\frac{q S+C N_{P} \beta r}{T S-C^{2} N_{A} N_{P} \alpha \beta} \\
& P^{*}=\frac{r T+C N_{A} \alpha q}{T S-C^{2} N_{A} N_{P} \alpha \beta}
\end{aligned}
$$

We note that any solution is biologically feasible only for $A^{*}$ and $P^{*}$ posi- 
tives. Bellow, we develop this point in detail for mutualistic and antagonistic networks. We chose to drop the \pm symbol in the last equation, instead we adopt $\alpha>0$ for mutualistic interactions and $\alpha<0$ for herbivory.

\subsection{Discussion of a Nonlinear Model}

We may also consider more sophisticated mutualistic models that take into account a non-linear saturation process as in the reference [19] [20]. We remark that the nonlinear model, as any ordinary differential equation model, is a mean field approach; to expand our methodology to a non mean field regime we would have to explicitly include the space by using differential partial equations or cellular automata models [12]. The linear mutualistic model presents an explosive behavior that can be solved using saturation in the interaction response between animal-plant. In reference [20] the mutualistic interaction for one animal and one plant species $A$ and $P$ is modeled as:

$$
\left\{\begin{array}{l}
\frac{\mathrm{d} A}{\mathrm{~d} t}=q A-T A^{2}+\frac{\beta P A}{1+\beta h P} \\
\frac{\mathrm{d} P}{\mathrm{~d} t}=r P-S P^{2}+\frac{\alpha A P}{1+\alpha h A}
\end{array}\right.
$$

However, for low handling time $h$, the non-linear equations reduce to the standard linear model. The main point in model choice is perhaps: how far are the linear model predictions compared to the non-linear one. The result of reference [20] is interesting, as it shows that the critical points of the non-linear model are in a broader interval which include the linear one, and the two models coincide in the limit of small non-linearity. In addition, the analytical treatment of the non-linear model is mathematically cumbersome and less intuitive than the linear case. Moreover, the linear model allows us to explicitly make analytical inferences and to test them. Despite its simplification, the linear model produces sound theoretical results that can be compared with empirical data.

\section{Condition for Existence of the Mean Field Model}

In our work, we study network properties and analyze what the MMFM can tell about network patterns that produce viable communities. In this way, we do not focus on species parameter $q, r, S$ and $T$ but on the interaction network properties: $\alpha$ and $\beta$ at one side and network parameters: $N_{A}, N_{P}$ and C.

We distinguish two situations in the study: the mutualistic interactions and the antagonistic interactions. The first is characterized by a positive effect of the plant animal encounter on plant population growth $\alpha>0$ whereas the second shows the opposite tendency that plant-animal interactions decrease plant growth $\alpha<0$. In the following, we distinguish these two situations to study MMFM conditions for the existence of viable solutions.

\subsection{Mutualism}

The condition for non-trivial existence of the system formed by Equations (7) 
and (8) assumes that both $A^{*}$ and $P^{*}$ should be positive. Since the numerator of the right side of equation is always positive, in order to fulfill both conditions it is necessary that the denominator will be positive. Then, we have:

$$
\alpha \beta<\frac{T S}{C^{2} N_{A} N_{P}} .
$$

This inequality imposes that the quantities $N_{A}, N_{P}, C, \alpha$ or $\beta$ should be small. The condition of small product $N_{A} \times N_{P}$ and connectance $C$ is related to sparsity and it is a well known property of ecological networks [15] [22]. Moreover, the condition of small product $\alpha \beta$ was explored in reference [28], where it is interpreted as the effect of asymmetric dependence in mutualistic networks. The limitations of the linear approach in this context was discussed in reference [20].

\subsection{Antagonism}

In the case of antagonistic interactions, we perform the transformation $\alpha \rightarrow-\alpha$. In this new situation, the denominator of Equations (7) and (8) will always be positive, and as a consequence, Equation (7) is trivially positive. The fixed point related to Equation (8) still has an additional condition that should be fulfilled:

$$
r T-C N_{A} \alpha q>0 .
$$

This new condition imposed to antagonistic systems implies that $C N_{A}$ should be small, which means that the overall number of links in an antagonistic web should not be large. The simplest way to guarantee this condition is that the number of animals in the web, $N_{A}$, should be small. We call attention that this condition is not present in mutualistic networks, therefore antagonistic webs should have a comparatively small number of animal species. We compare the number of animal and plant species for mutualistic and antagonistic cases in the next subsection.

\subsection{Data Comparison}

The condition for existence (11) which is exclusive of antagonistic networks claims that $N_{A}$ should be small. In this way, we expect that the number of animals in antagonistic networks should be more restricted than the number of plants.

The relative difference between animals and plants is presented in Figure 1. In this picture we show a bar-plot comparing the ratio $N_{A} / N_{P}$ for all pollinators and herbivorous webs. In addition, we performed the Student's $t$-test to check for difference between the groups $t=5.94, d f=46, p<0.0001$. We performed an extra test to check if the number of animals for herbivorous data set is significantly distinct from the number of plants $t=-0.38, d f=22, p=0.71$, which gives a negative result.

\section{Discussion}

In this paper, we work a multidimensional dynamic model that encompasses 


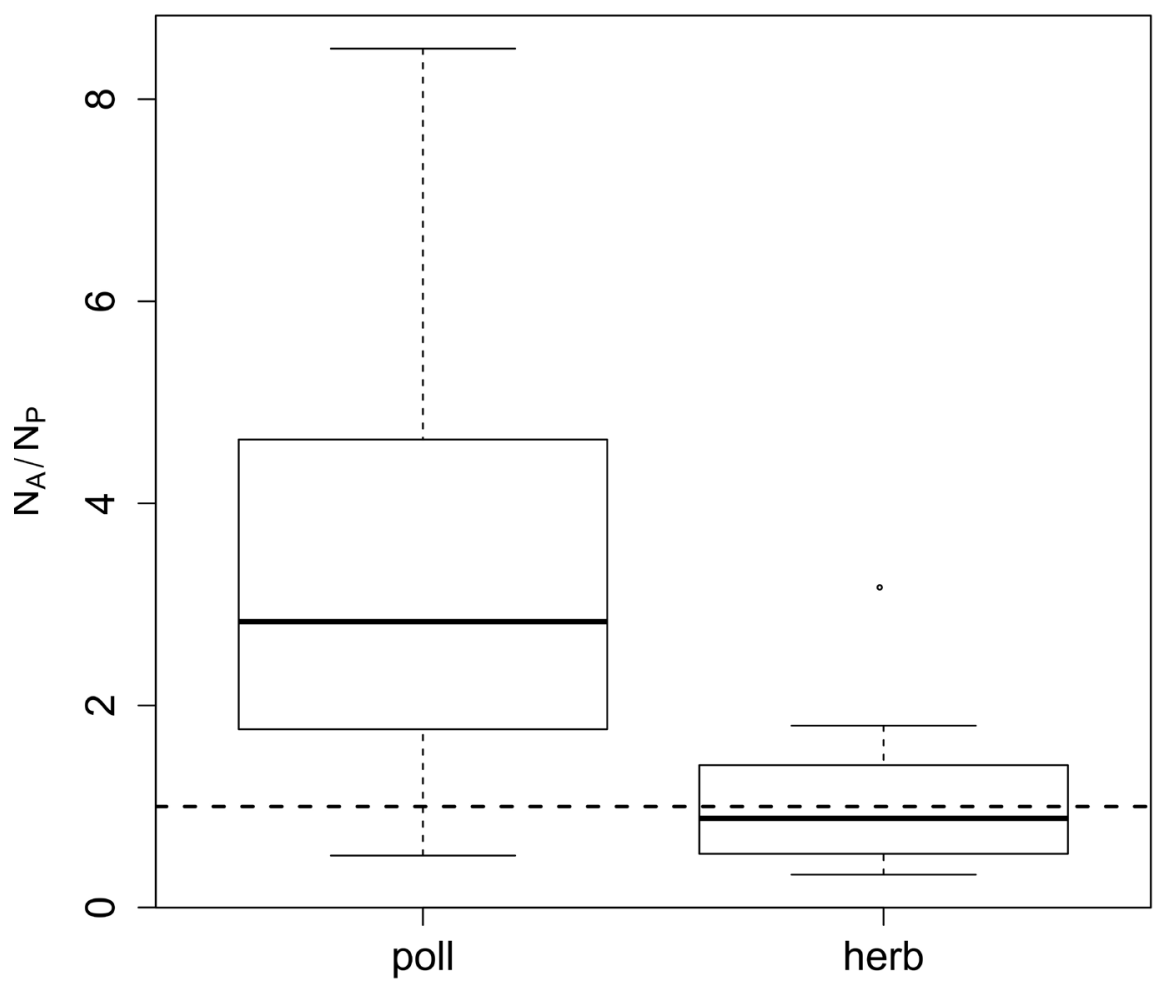

Figure 1. The figure shows the ratio $N_{A} / N_{P}$, the number of animal species divided by plant species in the network. The figure shows a larger relative number of animals in pollinator networks as compared to herbivorous networks. We depict a constant dashed line $N_{A} / N_{P}=1$ to help the visualization.

mutualistic and antagonistic interactions in a single framework. The MMFM is based on reducing a set of $N_{A}+N_{P}$ coupled equations to two equations that describe the dynamic evolution of a paradigmatic animal and plant of the community. At one hand, the MMFM implicates in a loss of information of the individual species and their interactions. On the other hand, the MMFM allows for computing the existence conditions of the system, and as a consequence, to make inference about these conditions of the full multispecies interaction network.

The essence of Mean Field approach in theoretical science consists in erasing individual differences to capture overall features of the system. In a mean field approximation, the interaction details among individuals in a network are less important than the average properties of the system dynamics. The challenge of the Mean Field Theory consists in not taking into account superfluous heterogeneities of the problem, but instead focusing on overall aspects of the phenomenon. The objective of the Mean Field theory is neither to give answers to individual differences, nor to look at small differences in the system. In this perspective, the MMFM still gives answers that can be empirically verified, despite obvious limitations and strong reductionism. The dependence asymmetry and the sparsity of the networks are positive results of the Mean Field approach and have already been discussed in the literature [26]. The difference between animal and plant species has not previously explored, and our work is the first to point 
out this theoretical result of the MMFM. In summary, the Mean Field Theory should be judged a posteriori. If the theoretical model gives a positive answer to empirical data, only then can it be taken seriously.

The idea that antagonistic interactions are more modular like, whereas mutualistic ones are more nested was extensively explored in the literature [15] [30]. The antagonistic relations tend to follow an arms race co-evolutionary strategy that produces a formation of cliques in the network, while the mutualistic interactions point to a generalist plus sub-generalist co-evolutionary strategy [31]. Our manuscript works out another, not so explored, difference between antagonistic and mutualistic networks: the number of animal and plants in their composition. In fact, an alternative and appealing title for our paper could be: "Why pollinator networks have more animals than plants in their structures than herbivorous networks?" This paper essays an explanation for this question, in fact, this is the objective of our work.

It is well known that biodiversity of animal species is larger than plant species [32] [33] [34]. Following this simple rule, we should generally expect that the number of animal species should surpass the number of plant species in interacting networks. This expectation could be used as a null model for plant and animal species occupancy in an interacting network. Our theoretical results suggest that it is not valid for antagonistic interaction networks, which is in agreement with empirical results. In this way, as stated in Figure 1, pollinator networks are closer to a null model of animal and plant occupancy than antagonistic networks which present a strong misbalance in their composition.

Finally, we believe that this work is important for the discussion of the complexity stability debate. We are conscious of the strong simplification of our mathematical framework. Initially we propose a model of coupled differential equations that is already a mean field approach because it does not take into account the spatial structure of the landscape. Over this model we proceed to perform a further simplification, reducing the number of variables from $N_{A}+N_{P}$ to 2. In this way, our theoretic approach proceeds over a double mean field approximation: first, we exclude the spatiality, and secondly we reduce the number of degrees of freedom. A comparison of our results with empirical data suggest that despite the strong simplification of our theoretical approach, the mean field strategy is indeed a valuable tool.

\section{Acknowledgements}

Financial support to Gilberto Corso from CNPq (Conselho Nacional de Desenvolvimento Científico e Tecnológico) is acknowledged.

\section{References}

[1] May, R.M. (1972) Will a Large Complex System Be Stable? Nature, 238, 413-414. https://doi.org/10.1038/238413a0

[2] May, R.M. (1974) Stability and Complexity in Model Ecosystems. Princeton University Press, Princeton. 
[3] Bastolla, U., Fortuna, M.A., Pascual-García, A., Ferrera, A., Luque, B. and Bascompte, J. (2009) The Architecture of Mutualistic Networks Minimizes Competition and Increases Biodiversity. Nature, 458, 1018-1020. https://doi.org/10.1038/nature07950

[4] Case, T.J. (2000) An Illustrated Guide to Theoretical Ecology. Oxford University Press, Oxford.

[5] Bastolla, U., Lässig, M., Manrubia, S.C. and Valleriani, A. (2005) Biodiversity in Model Ecosystems, I: Coexistence Conditions for Competing Species. Journal of Theoretical Biology, 235, 521-530. https://doi.org/10.1016/j.jtbi.2005.02.005

[6] James, A., Pitchford, J.W. and Plank, M.J. (2012) Disentangling Nestedness Disentangled. Nature, 487, 227-230. https://doi.org/10.1038/nature11214

[7] Lässig, M., Bastolla, U., Manrubia, S.C. and Valleriani, A. (2001) Shape of Ecological Networks. Physical Review Letters, 86, 4418-4421. https://doi.org/10.1103/PhysRevLett.86.4418

[8] Strogatz, S.H. (2001) Exploring Complex Networks. Nature, 410, 268-276. https://doi.org/10.1038/35065725

[9] Newman, M.E.J. (2003) The Structure and Function of Complex Networks. SIAM Review, 45, 167-256. https://doi.org/10.1137/S003614450342480

[10] Ings, T.C., Montoya, J.M., Bascompte, J., Blüthgen, N., Brown, L., Dormann, C.F., et al. (2009) Ecological Networks-Beyond Food Webs. Journal of Animal Ecology, 78, 253-269. https://doi.org/10.1111/j.1365-2656.2008.01460.x

[11] Albert, R. and Barabási, A.-L. (2002) Statistical Mechanics of Complex Networks. Reviews of Modern Physics, 74, 47-98. https://doi.org/10.1103/RevModPhys.74.47

[12] Solé, R.V. and Bascompte, J. (2006) Self-Organization in Complex Ecosystems. Princeton University Press, Princeton.

[13] Bascompte, J. (2009) Disentangling the Web of Life. Science, 325, 416-419. https://doi.org/10.1126/science.1170749

[14] Guimarães, P.R., Raimundo, R.L.G. and Cagnolo, L. (2015) Interaction Web Database. National Center for Ecological Analysis and Synthesis. https://www.nceas.ucsb.edu/interactionweb/index.html

[15] Bascompte, J. and Jordano, P. (2013) Mutualistic Networks. Princeton University Press, Princeton. https://doi.org/10.1515/9781400848720

[16] Vázquez, D.P., Poulin, R., Krasnov, B.R. and Shenbrot, G.I. (2005) Species Abundance and the Distribution of Specialization in HOST-PARASITE Interaction Networks. Journal of Animal Ecology, 74, 946-955. https://doi.org/10.1111/j.1365-2656.2005.00992.x

[17] Lewinsohn, T.M., Novotny, V. and Basset, Y. (2005) Insects on Plants: Diversity on Herbivore Assemblages Revisited. Annual Review of Ecology, Evolution, and Systematics, 36, 597-620. https://doi.org/10.1146/annurev.ecolsys.36.091704.175520

[18] Alligood, K.T., Sauer, T.D. and Yorke, J.A. (1997) Chaos: An Introduction to Dynamical Systems. Springer, New York. https://doi.org/10.1007/978-3-642-59281-2

[19] Holland, J.N., DeAngelis, D.L. and Bronstein, J.L. (2002) Population Dynamics and Mutualism: Functional Responses of Benefits and Costs. The American Naturalist, 159, 231-244. https://doi.org/10.1086/338510

[20] Holland, J.N., Okuyama, T. and DeAngelis, D.L. (2006) Comment on “Asymmetric Coevolutionary Networks Facilitate Biodiversity Maintenance”. Science, 313, 1887. https://doi.org/10.1126/science.1129547

[21] MacArthur, R.H. (1972) Geographical Ecology. Princeton University Press, Prince- 
ton.

[22] Jordano, P. (1987) Patterns of Mutualistic Interactions in Pollination and Seed Dispersal: Connectance, Dependence Asymmetries, and Coevolution. American Naturalist, 129, 657-677. https://doi.org/10.1086/284665

[23] Memmott, J. (1999) The Structure of a Plant-Pollinator Food Web. Ecology Letters, 2, 276-280. https://doi.org/10.1046/j.1461-0248.1999.00087.x

[24] Nascimento, A.R., Neto, M.A., Almeida, A.M., Fonseca, C.R., Lewinsohn, T.M. and Penteado-Dias, A. (2014) Parasitoid Wasps in Flower Heads of Asteraceae in the Brazilian Cerrado: Taxonomical Composition and Determinants of Diversity. Neotropical Entomology, 43, 298-306. https://doi.org/10.1007/s13744-014-0218-9

[25] Corso, G., Cruz, C.P.T., Pinto, M.P., de Almeida, A.M. and Lewinsohn, T.M. (2015) Binary versus Weighted Interaction Networks. Ecological Complexity, 23, 68-72. https://doi.org/10.1016/j.ecocom.2015.04.003

[26] Bascompte, J., Jordano, P. and Olesen, J.M. (2006) Asymmetric Coevolutionary Networks Facilitate Biodiversity Maintenance. Science, 312, 431-433.

https://doi.org/10.1126/science.1123412

[27] Murray, J.D. (2002) Mathematical Biology: An Introduction. Springer-Verlag, Berlin.

[28] Goldenfeld, N. (1992) Lectures on Phase Transitions and the Renormalization Group (Frontiers in Physics). Addison-Wesley, Boston.

[29] Marro, J. and Dickmann, R. (1999) Nonequilibrium Phase Transitions in Lattice Models. Cambridge University Press, Cambridge.

https://doi.org/10.1017/CBO9780511524288

[30] Thébault, E. and Fontaine, C. (2008) Does Asymmetric Specialisation Differ between Mutualistic and Trophic Networks? Oikos, 117, 555-563. https://doi.org/10.1111/j.0030-1299.2008.16485.x

[31] Thébault, E. and Fontaine, C. (2010) Stability of Ecological Communities and the Architecture of Mutualistic and Trophic Networks. Science, 329, 853-856. https://doi.org/10.1126/science.1188321

[32] May, R.M. and Beverton, R.J.H. (1990) How Many Species? Philosophical Transactions of the Royal Society B, 330, 293-304. https://doi.org/10.1098/rstb.1990.0200

[33] Wilson, E.O. (1999) The Diversity of Life. W. W. Norton \& Company, New York.

[34] Mora, C., Tittensor, D.P., Adl, S., Simpson, A.G.B. and Worm, B. (2011) How Many Species Are There on Earth and in the Ocean? PLoS ONE, 9, e1001127. https://doi.org/10.1371/journal.pbio.1001127 
Submit or recommend next manuscript to SCIRP and we will provide best service for you:

Accepting pre-submission inquiries through Email, Facebook, LinkedIn, Twitter, etc. A wide selection of journals (inclusive of 9 subjects, more than 200 journals)

Providing 24-hour high-quality service

User-friendly online submission system

Fair and swift peer-review system

Efficient typesetting and proofreading procedure

Display of the result of downloads and visits, as well as the number of cited articles Maximum dissemination of your research work

Submit your manuscript at: http://papersubmission.scirp.org/

Or contact oje@scirp.org 